\title{
Optimization of multiple-layer microperforated panels by simulated annealing
}

\author{
Heidi Ruiz $^{\mathrm{a}, *}$, Pedro Cobo ${ }^{\mathrm{a}}$, Finn Jacobsen ${ }^{\mathrm{b}}$ \\ ${ }^{a}$ Center for Applied Acoustics and Non Destructive Testing CAEND, CSIC-UPM, Serrano 144, 28006 Madrid, Spain \\ ${ }^{\mathrm{b}}$ Acoustic Technology, Department of Electrical Engineering, Technical University of Denmark, Building 352, Ørsteds Plads, DK-2800 Kgs. Lyngby, Denmark
}

\section{A R T I C L E I N F O}

\section{Article history:}

Received 5 April 2011

Received in revised form 15 April 2011

Accepted 20 April 2011

Available online 11 May 2011

\section{Keywords:}

Sound absorption

Microperforated panels

Simulated annealing

\begin{abstract}
A B S T R A C T
Sound absorption by microperforated panels (MPP) has received increasing attention the past years as an alternative to conventional porous absorbers in applications with special cleanliness and health requirements. The absorption curve of an MPP depends on four parameters: the holes diameter, the panel thickness, the perforation ratio, and the thickness of the air cavity between the panel and an impervious wall. It is possible to find a proper combination of these parameters that provides an MPP absorbing in one octave band or two, within the frequency range of interest for noise control applications. However, when a wider absorption frequency band is required, it is necessary to design multiple-layer MPP (ML-MPP). The design of an $N$-layers MPP depends on $4 \mathrm{~N}$ parameters. Consequently, the tuning of an optimal MLMPP by exhaustive search within a prescribed frequency band becomes impractical. Therefore, simulated annealing is proposed in this paper as a tool to solve the optimization problem of finding the best combination of the constitutive parameters of an ML-MPP providing the maximum average absorption within a prescribed frequency band.
\end{abstract}

() 2011 Elsevier Ltd. All rights reserved.

\section{Introduction}

Microperforated panels (MPP) provide wideband absorption without any porous materials. The absorption is produced by viscous losses in an array of sub-millimetric holes along the panel when it is mounted in front of an impervious wall, with an air cavity in between. The performance depends on the thickness of the panel, $t$, the hole diameter, $d$, the porosity, $p$, and the thickness of the air cavity, $D$. They were first proposed by Maa [1,2] and further used for different applications [3,4]. MPPs are resonator type absorbers. Therefore, single-layer MPPs have a frequency band of absorption that usually amounts to as much as 2.5 octaves. It is possible to widen the absorption band without decreasing the curve peak by designing multiple-layer MPPs [4-9]. Since the performance of a single-layer MPP depends on four parameters $(d, t, p, D)$, the design of an $N$-layer MPP will involve the tuning of $4 N$ parameters.

From an engineering point of view, it would be attractive to know the combination of constitutive parameters within given variation ranges that provides the maximum mean absorption for a prescribed frequency band. This is a typical optimization problem. More specifically, it consists of looking for a global maximum in a solutions space where there may be a number of local maxima. Simulated annealing (SA) is an optimization method especially appropriate for such kind of problems $[10,11]$. The SA algorithm

\footnotetext{
* Corresponding author.

E-mail address: heidiruizv@gmail.com (H. Ruiz).
}

has recently been successfully applied to a number of acoustical problems, such as the optimization of constrained composite absorbers [12], noise barriers [13,14] and noise reduction algorithms [15]. Kim and Kim [16] studied a similar problem related to the optimization of a multilayer system composed of solid panels, perforated panels and air gaps. However, while these authors kept fixed the constitutive parameters of the panels and optimized the layer sequence and thickness of each layer simultaneously using topology optimization, we propose the application of SA for optimizing the mean absorption of ML-MPPs, within given variation ranges of their constitutive parameters.

The paper is organized as follows. First, the equations for the normal incidence absorption coefficient of ML-MPPs are presented in Section 2. The SA algorithm to optimize the mean absorption within a prescribed frequency band is described in Section 3. Next, experimental results of absorption that validate the theory are presented in Section 4. Finally, Section 5 summarizes the main results of the investigation.

\section{Normal incidence sound absorption of an ML-MPP}

Fig. 1 shows a sketch of an ML-MPP composed of $N$ layers. The normal incidence absorption coefficient of a system is

$$
\alpha(f)=1-|R(f)|^{2}
$$

where 

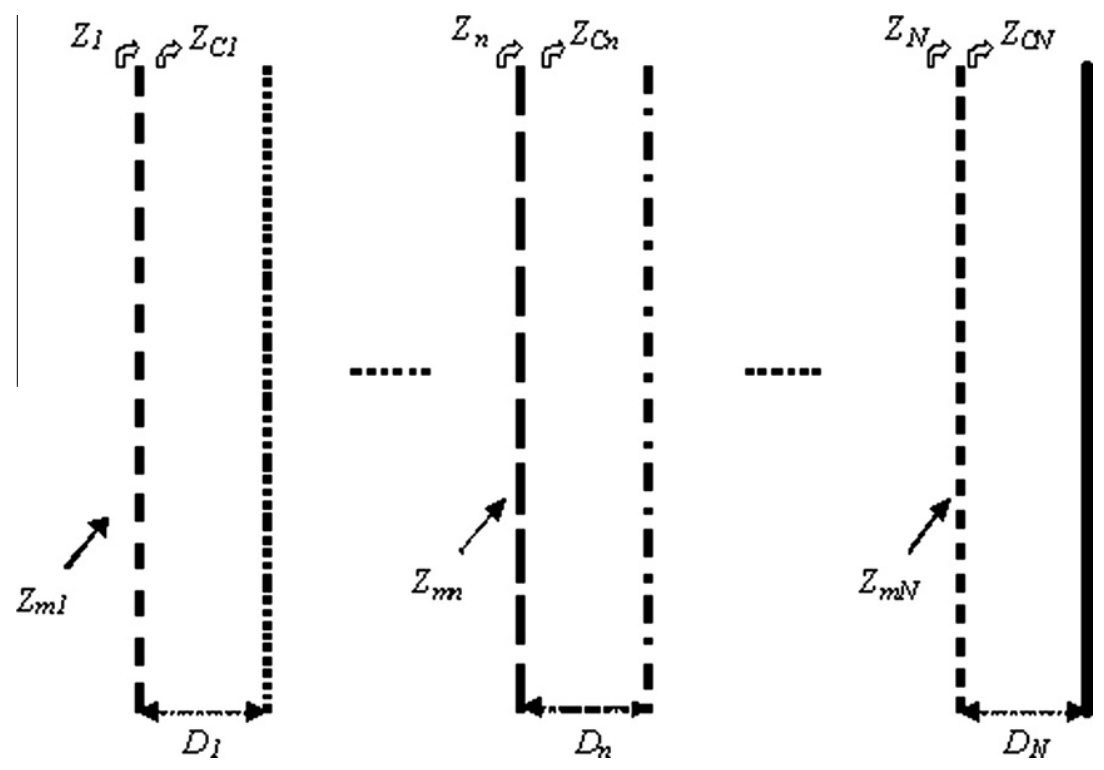

Fig. 1. Sketch of an ML-MPP.

$R(f)=\frac{Z_{i}(f)-Z_{0}}{Z_{i}(f)+Z_{0}}$

is the normal incident reflection coefficient, $Z_{i}$ is the input impedance, and $Z_{0}=\rho_{0} c_{0}$ is the characteristic impedance of air. The acoustic impedance at the input of the $n$th layer is [8].

$Z_{n}=Z_{m n}+Z_{C n}$,

where

$Z_{C n}=Z_{0} \frac{Z_{n+1} \cos \left(k D_{n}\right)+j Z_{0} \sin \left(k D_{n}\right)}{Z_{0} \cos \left(k D_{n}\right)+j Z_{n+1} \sin \left(k D_{n}\right)}$

is the acoustic impedance of the $n$th cavity [17] of thickness $D_{n}, Z_{m n}$ is the acoustic impedance of the $n$th MPP, and $k$ is the wavenumber. Eq. (4) applies for $n=1,2, \ldots, N-1$. For $n=N$,

$Z_{C N}=-j Z_{0} \cot \left(k D_{N}\right)$.

Eqs. (1)-(5) make it possible to obtain the absorption coefficient of an $N$-layers MPP provided that the equation for the acoustic impedance of an MPP is known. Maa [2] proposed his well-known equation based on the Crandall solution for the impedance of a tube with the edge corrections of Ingard,

$$
\begin{aligned}
Z_{m n, \text { Maa }}= & \frac{\sqrt{2} \mu y_{n}}{p_{n} d_{n}} \\
& +\frac{j \omega \rho_{0}}{p_{n}}\left\{0.85 d_{n}+t_{n}\left[1-\frac{2 J_{1}\left(y_{n} \sqrt{-j}\right)}{\left(y_{n} \sqrt{-j} J_{0}\left(y_{n} \sqrt{-j}\right)\right)}\right]^{-1}\right\},
\end{aligned}
$$

where $\left(d_{n}, t_{n}, p_{n}\right)$ are the hole diameter, thickness and porosity, respectively, of the $n$th MPP, $\mu$ is the air viscosity coefficient, $\rho_{0}$ the air density, $\omega$ the angular frequency, $J_{0}, J_{1}$ are Bessel functions of the first kind and order 0 and 1, respectively, and

$y_{n}=d_{n} / d_{v}=d_{n} \sqrt{\rho_{0} \omega / 4 \mu}$,

is the ratio of the hole diameter to the viscous layer thickness $\left(d_{v}\right)$.

Eq. (6) is correct for an MPP with a low perforation ratio. When the holes are too close, it is necessary to introduce a correction to the reactance term of the end correction to take the interaction between holes into account [18]. This is done by including the Fok function into the denominator of the reactive term of the edge correction in Eq. (6),

$$
\begin{aligned}
\psi\left(v_{n}\right)= & 1 /\left(1-1.4092\left(v_{n}\right)+0.33818\left(v_{n}\right)^{3}+0.06793\left(v_{n}\right)^{5}\right. \\
& \left.-0.02287\left(v_{n}\right)^{6}+0.03015\left(v_{n}\right)^{7}-0.01641\left(v_{n}\right)^{8}\right)
\end{aligned}
$$

with

$v_{n}=\sqrt{p_{n}}$.

More recently, Atalla and Sgard [19] proposed an alternative equation valid for microperforated panels and screens,

$Z_{m n, A S}=\left(t_{n}+2 \varepsilon_{e n}\right)\left[(1+j) \frac{4 R_{s}}{p_{n} d_{n}}+\frac{j \omega \rho_{0}}{p_{n}}\right]$,

where $\varepsilon_{e n}=0.425 d_{n}\left(1-1.14 \sqrt{p_{n}}\right)$ is the length correction, and $R_{s}=\sqrt{\rho_{0} \omega \mu / 2}$ is the surface resistance, of the air vibrating within each hole of the MPP.

Whereas Eq. (6) was originally proposed for circular holes, Eq. (9) presents a general methodology that can handle particular geometrical parameters for miscellaneous configurations [19]. The perforations must be sub-millimetric to provide an MPP with enough absorption in the frequency band of interest in noise control applications. Sub-millimetric perforations are usually carried out by laser technology. Numerous circular punctures must be made even for moderate porosities, and this can become rather expensive. The manufacturing process will be cheaper if slit perforations are made instead of circular holes. Specific impedance models were proposed for micro-slotted panels (MSP), e.g. by Maa [20] and Randeberg [21].

Fig. 2 shows the absorption curves of a single-layer micro-slotted panel with parameters $(d, t, p, D)=(0.25 \mathrm{~mm}, 1 \mathrm{~mm}, 3.4 \%, 1.1 \mathrm{~cm})$ predicted using the equations of Maa [2], Atalla and Sgard [19], Maa [20] and Randeberg [21]. Here $d$ is the width of the slits (i.e., the hydraulic diameter). For the sake of comparison, the experimental curve measured in an impedance tube by the transfer function method [22] is also shown. As can be seen, the absorption curves vary for the four models. Of those presented, the curve provided by the Atalla and Sgard model agrees best with the experimental results. Therefore, we will use Eq. (9) in the following for modeling the absorption of an ML-MPP. 


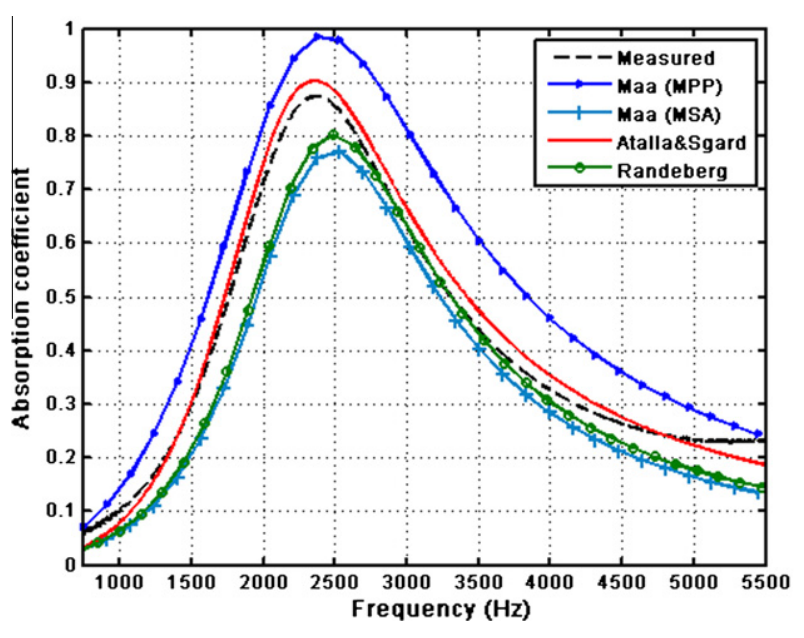

Fig. 2. Absorption curves of a single-layer MPP with parameters $(d, t, p, D)=(0.25 \mathrm{~mm}, 1 \mathrm{~mm}, 3.4 \%, 1.1 \mathrm{~cm})$.

\section{Optimization by simulated annealing}

The problem set forth is to find the best combination of constitutive parameters of an ML-MPP that provides the maximum mean absorption, within a prescribed frequency band. Such parameters should be confined to take values in constrained ranges.

To illustrate the problem, Fig. 3 shows the mean absorption of a double-layer MPP (DL-MPP), in the frequency band $(800,6400) \mathrm{Hz}$ (three octaves), as a function of the configuration number [each configuration is a combination of the six parameters $\left.\left(d_{1}, p_{1}, D_{1}, d_{2}, p_{2}, D_{2}\right)\right]$. The number of configurations $\left(10^{6}\right.$, in this case) has been taken purposely low so that the problem can be solved by exhaustive search (ES).

Note that the objective function (average absorption) has a spiky structure, with a global maximum and many local maxima. Classical optimization techniques, based on the calculation of derivatives, are precluded in these problems, since they have the risk to be trapped in a local maximum. Conversely, SA is a technique especially well suited for solving global optimization problems with many local optima.

SA mimics the way metals cool and anneal [23]. If the metal is slowly cooled from a high temperature state, atoms are rearranged

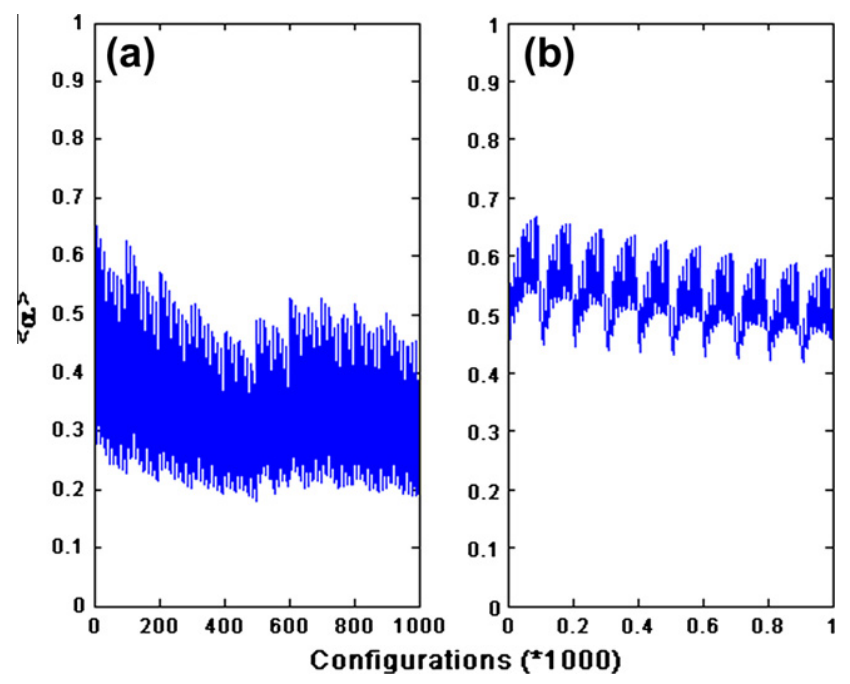

Fig. 3. (a) Evolution of the average absorption with the configuration number for the case of exhaustive search. (b) A zoom of (a). to attain minimum energy, following the Boltzmann law, which asserts that the probability that the system evolves from one state to another with energy change $\Delta E$ at temperature $T$ is $\exp \left(-\Delta E / k_{B} T\right)$, $k_{B}$ being the Boltzmann constant. The application of this scheme of accepting a new state, or configuration, to the function optimization problem is known as the Metropolis algorithm [24]. To apply this algorithm to an optimization problem, it is necessary to provide the following elements [23]: (a) the space of configurations; (b) a random mechanism for changing from one configuration to other; (c) an objective function (analogous to energy) to be optimized; (d) a control parameter (analogous to temperature) to start and finish the algorithm; and (e) a cooling schedule, controlled by a cooling factor, i.e., a constant, that establishes how the control parameter is lowered from high to low values.

In the present case, the space of configurations is given by the combinations of constitutive parameters, with the constrains

$$
\begin{aligned}
& d_{1,2} \in\left[d_{\min }, d_{\max }\right], \\
& p_{1,2} \in\left[p_{\min }, p_{\max }\right], \\
& D_{1,2} \in\left[D_{\min }, D_{\max }\right],
\end{aligned}
$$

keeping $t_{1}=t_{2}=1 \mathrm{~mm}$. To pass from one configuration to another, one parameter, e.g., $\left(d_{1,2}, p_{1,2}\right)$ or $\left(D_{1,2}\right)$, is chosen and varied randomly within the constrained range. This mechanism guarantees that two successive configurations are in the neighborhood of each other. For each configuration the Metropolis algorithm is implemented in the following manner. Let

$\Delta \alpha\left(T_{n}\right)=\left\langle\alpha_{\text {old }}\right\rangle-\left\langle\alpha_{\text {new }}\right\rangle$

where $\langle\alpha\rangle$ denotes the average absorption in the frequency band $\left(f_{1}, f_{2}\right)$ and $T_{n}$ is the control parameter. Two conditions are used to decide whether or not the configuration is accepted: (a) $\Delta \alpha\left(T_{n}\right)<0$, or (b) the acceptance probability, $P\left(T_{n}\right)=\exp \left(-\Delta \alpha\left(T_{n}\right) / T_{n}\right)>\varphi$ where $\varphi$ is a random number generated in the interval $[0,1]$. This means that configurations with larger average absorption are accepted unconditionally, while configurations with lesser average absorption are accepted only if the Boltzmann function value is larger than $\varphi$. This mechanism prevents the search from being trapped in a local maximum.

As stated above, the objective function to be maximized is the average absorption in the frequency band $\left(f_{1}, f_{2}\right)$. The control parameter $T_{n}$, which varies from $T_{\text {ini }}$ to $T_{\text {end }}$, cools as
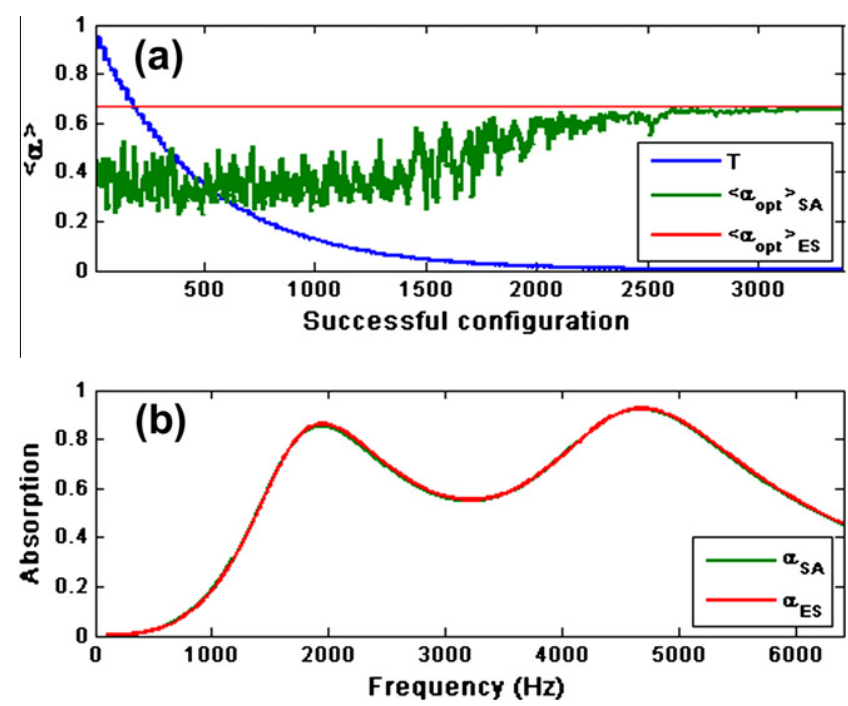

Fig. 4. (a) Evolution of temperature and objective function of $S A$, and (b) absorption curves obtained by SA and ES, for the case of a DL-MPP, with $\left(f_{1}, f_{2}\right)=(800,6400) \mathrm{Hz}$, $T_{\text {ini }}=1, T_{\text {end }}=0.0001, \beta=0.95, N_{\text {iter }}=250, N_{\text {success }}=25$. 
Table 1

Constitutive parameters and average absorption of a DL-MPP with $t_{1}=t_{2}=1 \mathrm{~mm}$ found out by ES and SA.

\begin{tabular}{|c|c|c|c|c|c|c|c|}
\hline \multirow[t]{2}{*}{ Algorithm } & \multicolumn{3}{|l|}{ MPP1 } & \multicolumn{3}{|l|}{ MPP2 } & \multirow[t]{2}{*}{$\langle\alpha\rangle$} \\
\hline & $\begin{array}{l}d_{1} \\
(\mathrm{~mm})\end{array}$ & $\begin{array}{l}p_{1} \\
(\%)\end{array}$ & $\begin{array}{l}D_{1} \\
(\mathrm{~cm})\end{array}$ & $\begin{array}{l}d_{2} \\
(\mu \mathrm{m})\end{array}$ & $\begin{array}{l}p_{2} \\
(\%)\end{array}$ & $\begin{array}{l}D_{2} \\
(\mathrm{~cm})\end{array}$ & \\
\hline ES & 0.25 & 8.5 & 1.0 & 0.25 & 3.4 & 1.0 & 0.6662 \\
\hline SA & 0.25 & 8.4 & 1.0 & 0.25 & 3.5 & 1.0 & 0.6582 \\
\hline
\end{tabular}
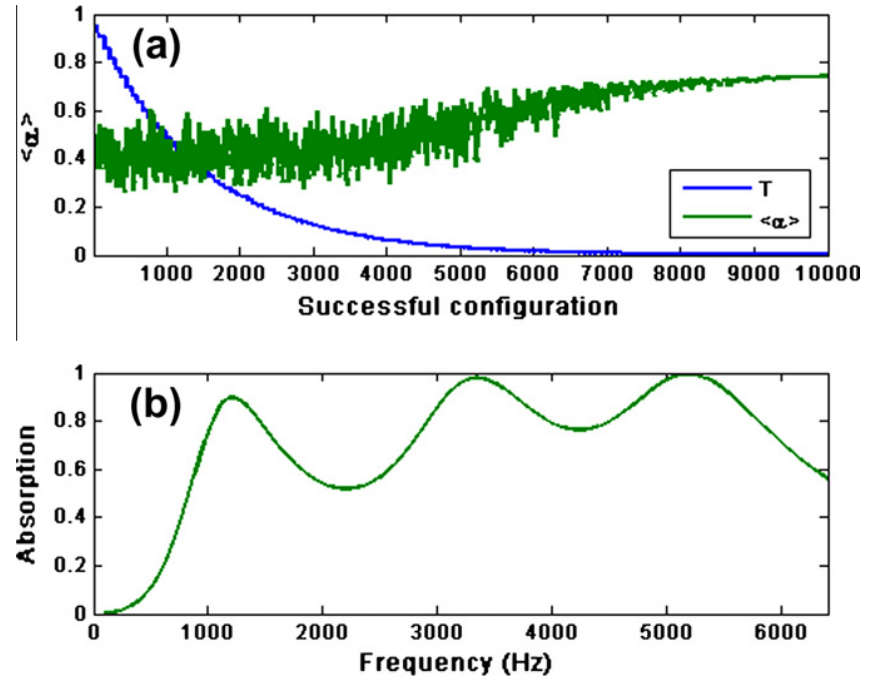

Fig. 5. (a) Evolution of temperature and objective function of SA, and (b) absorption curves obtained by SA and ES, for the case of a TL-MPP, with $\left(f_{1}, f_{2}\right)=(400,6400) \mathrm{Hz}$, $T_{\text {ini }}=1, T_{\text {end }}=0.00001, \beta=0.95, N_{\text {iter }}=500, N_{\text {success }}=75$.

$T_{n}=\beta T_{n+1}$

where $\beta$ is the cooling factor. The system progresses from $T_{n}$ to $T_{n+1}$ when a number of iterations, $N_{i t e r}$, are completed. The process is finished when either $T_{\text {end }}$ is reached or a number of changes, $N_{\text {success }}$, are done without success in finding a better configuration.

For the sake of comparison, the DL-MPP optimization problem worked out above by ES is now solved by simulated annealing. The constrained ranges for searching the constitutive parameters of the panels have been $d_{1,2} \epsilon[0.25,0.75] \mathrm{mm}, t_{1,2}=1 \mathrm{~mm}$, $p_{1,2} \epsilon[3.4,8.5] \%, D_{1,2} \in[1,5] \mathrm{cm}$. Fig. 4a illustrates the evolution of the temperature and objective function (the average absorption)

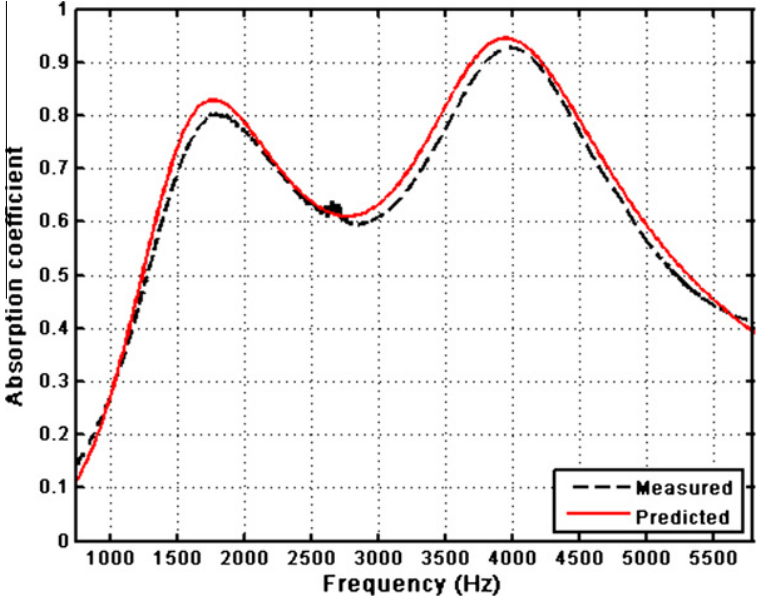

Fig. 7. Absorption curves of a DL-MSP with $\left(d_{1}, t_{1}, p_{1}, D_{1}, d_{2}, t_{2}, p_{2}, D_{2}\right)=(0.25 \mathrm{~mm}, 1 \mathrm{~mm}, 8.4 \%, 1 \mathrm{~cm}, 0.25 \mathrm{~mm}, 1 \mathrm{~mm}, 3.5 \%, 1 \mathrm{~cm})$.

of the algorithm. Initially, at a high temperature, there is a high probability of accepting a configuration with less average absorption. However, when the temperature decreases the probability of accepting configurations with less average absorption becomes increasingly smaller, until it finally converges to the optimum solution with an average absorption close to the maximum. Fig. 4b shows the absorption curve of the corresponding DL-MPP achieved by SA, as compared to the global optimum found by ES. The two curves are practically undistinguishable, for the plot resolution.

Table 1 summarizes the optimum parameters found by ES and SA. As can be seen, the porosities obtained by the two methods vary slightly from each other. The SA optimization results in an average absorption that differs just $1 \%$ with respect to the global optimum provided by ES. However, whereas the ES requires $3227 \mathrm{~s}$ to find the solution, the SA algorithm spent less than $16 \mathrm{~s}$ on providing the solution in an Intel Core i3 personal computer.

Let us calculate now the optimum triple-layer MPP (TL-MPP) for a wider frequency band $(400,6400) \mathrm{Hz}$ (four-octaves). In this case, keeping $t_{1}=t_{2}=t_{3}=1 \mathrm{~mm}$, there are 109 configurations to look for, so that the use of the exhaustive search is prohibitive. However, simulated annealing provides a solution close to the optimum in $68 \mathrm{~s}$, see Fig. 5 . Note that the average absorption converges to the optimum when the temperature colds enough, Fig. 5a. The SA algorithm provides the optimum TL-MPP with parameters $\left(d_{1}, t_{1}, p_{1}, D_{1}, d_{2}, t_{2}, p_{2}, D_{2}, d_{3}, t_{3}, p_{3}, D_{3}\right)=(0.25 \mathrm{~mm}, 1 \mathrm{~mm}, 8.5 \%, 1 \mathrm{~cm}$, $0.25 \mathrm{~mm}, 1 \mathrm{~mm}, 4,55 \%, 1 \mathrm{~cm}, 0.25 \mathrm{~mm}, 1 \mathrm{~mm}, 3.4 \%, 1.3 \mathrm{~cm})$ which
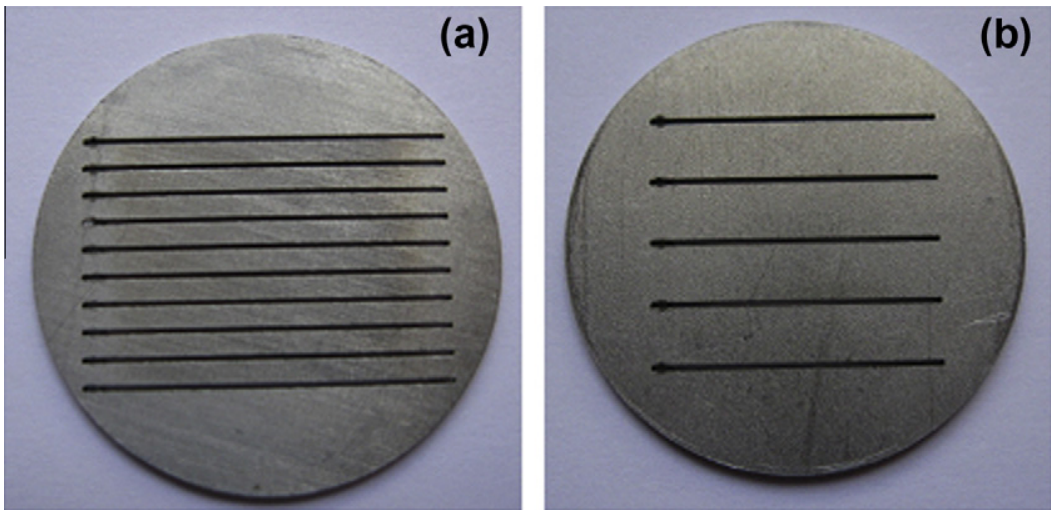

Fig. 6. MPPs used for experimental validation. (a) MPP1, (b) MPP2. 
gives an average absorption of $74.2 \%$ in the frequency band (400,6400) Hz (Fig. 5b).

\section{Experimental validation}

Measurements were performed for a DL-MPP inside an impedance tube with a diameter of $2.9 \mathrm{~cm}$. The samples were placed at one end of the tube and a loudspeaker radiating random noise was installed on the opposite side. The absorption coefficient was measured with the transfer function method [22]. The distance between microphones was $2 \mathrm{~cm}$; this should be less than half a wavelength. The inner tube diameter was $29 \mathrm{~mm}$, and therefore the valid frequency range for the measurement was $857 \mathrm{~Hz}<f<$ $6860 \mathrm{~Hz}$ [25]. Fig. 6 shows the MPP's used for the experiment with the constitutive parameters provided by the SA algorithm (see Table 1).

Fig. 7 shows a comparison between the experimental curve for the absorption as a function of the frequency and the simulation of the theoretical curve by Atalla and Sgard. The two curves approach each other. Slight differences between the curves can be due to small manufacturing imperfections (see Fig. 6) of the panels and their positioning inside the tube.

\section{Conclusions}

In this paper, the optimization of an ML-MPP design by simulated annealing has been presented. The absorption curve of a single-layer absorbing system is determined by four parameters: the constitutive parameters of the panel and the thickness of the air cavity. Furthermore, the absorption curve of an $\mathrm{N}$-layers system depends on $4 N$ parameters. For a double-layer system the exhaustive search technique is feasible but stops short for greater configurations due to very long processing time. Therefore, the SA algorithm is proposed as an effective, fast and straightforward technique to obtain the best possible configuration for an absorbing system composed of multiple layers. A study of the performance of the absorbers conditioned by their parameters shows a strong dependence mainly on the diameter of the holes and the porosity of the plate. The proposed double-layer prototype gives an average absorption of $66 \%$, for a frequency band up to $6400 \mathrm{~Hz}$. The optimization algorithm provided a solution in less than one minute, i.e., $5 \%$ of the time required by the exhaustive search technique. The predicted performance of the plate was validated in an impedance tube. Experimental measures show a very good agreement with the general model for microperforated plates proposed by Atalla and Sgard. A microperforated panel can be designed with slit perforations making the manufacture using laser techniques easier and cheaper.

\section{Acknowledgement}

This work has been supported by the Spanish Ministry of Science and Innovation, through Grant TRA2008-05654-C03-03.

\section{References}

[1] Maa DY. Microperforated-panel wideband absorbers. Noise Control Eng J 1987;29:77-84.

[2] Maa DY. Potential of microperforated panel absorber. J Acoust Soc Am 1998; 104:2861-6.

[3] Kang J, Fuchs HV. Predicting the absorption of open weave textiles and microperforated membranes backed by an air space. J Sound Vib 1999;220:905-20.

[4] Lee J, Swenson GW. Compact sound absorbers for low frequencies. Noise Control Eng J 1992;38:109-17.

[5] Lee DH, Kwon P. Estimation of the absorption performance of multiple layer perforated panel systems by transfer matrix method. J Sound Vib 2004;278:847-60.

[6] Sakagami K, Nakamori T, Morimoto M, Yairi M. Double-leaf microperforated panel space absorbers: a revised theory and detailed analysis. Appl Acoust 2009;70:703-9.

[7] Sakagami K, Morimoto M, Wakana K. A numerical study of double-leaf microperforated panel absorbers. Appl Acoust 2005;67:609-19.

[8] Cobo P, Cuesta M, Siguero M. Comparison of models describing double layer microperforated absorbers. Noise Control Eng J 2009;57:10-5.

[9] Cobo P, Ruiz H, Alvarez J. Double-layer microperforated panel/porous absorber as liner for anechoic closing of the test section in wind tunnels. Acta Acust United Ac 2010;96:914-22.

[10] Kirpatrick S, Gerlatt CD, Vecchi MP. Optimization by simulated annealing. Science 1983;220:671-80.

[11] Brooks SP, Morgan BJT. Optimization using simulated annealing. Statistiscian 1995;44:241-57.

[12] Chang YC, Yeh LJ, Chiu MC. Optimization of constrained composite absorbers using simulated annealing. Appl Acoust 2005;66:341-52.

[13] Mun S, Cho YH. Noise barrier optimization using a simulated annealing algorithm. Appl Acoust 2009;70:1094-8.

[14] Chiu MC. Optimization of equipment allocation and sound-barriers shape in a multi-noise plant by using simulated annealing. Noise Vib Worldwide 2009;40:23-35

[15] Bai MR, Hsieh PJ, Hur KN. Optimal design of minimum mean-square error noise reduction algorithms using the simulated annealing technique. J Acoust Soc Am 2008;125:934-43.

[16] Kim YJ, Kim YY. Solid-perforated panel layout optimization by topology optimization based on unified transfer matrix. J Acoust Soc Am 2010;4:1777-88.

[17] Brekhovskikh LM. Waves in layered media. New York: Academic Press; 1980

[18] Melling TH. The acoustic impedance of perforates at medium and high sound pressure levels. J Sound Vib 1973;125:1-65.

[19] Atalla N, Sgard F. Modeling of perforated plates and screens using rigid frame porous models. J Sound Vib 2007;303:195-208.

[20] Maa DY. Theory of microslit absorbers. Chinese J Acoust 2001;20:1-10.

[21] Randeberg RT. Adjustable slitted panel absorber. Acta Acust United Ac 2002;88:507-12.

[22] Chung JY, Blaser DA. Transfer function method of measuring in-duct acoustic properties. I. Theory. J Acoust Soc Am 1980;68(3):907-13.

[23] Press WH, Flannery BP, Teukolsky SA, Vetterling WT. Numerical recipes. The art of scientific computing. New York: Cambridge University Press; 1986.

[24] Metropolis N, Rosenbluth AW, Rosenbluth MN, Teller AH. Equations of state calculations by fast computing machines. J Chem Phys 1953;21:1087-92.

[25] ISO 10534-2. Determination of sound absorption coefficient and impedance in impedance tubes. Part 2: transfer-function method; 1998. 